\section{ADVANCED MATERIALS TECHNOLOGIES}

PREPRINT OF ARTICLE PUBLISHED IN ADVANCED MATERIAL TECHNOLOGIES OFFICIAL VERSION CAN BE OBTAINED AT https://doi.org/10.1002/admt.201700102

DOI: $10.1002 / \mathrm{admt} .201700102$

Article type: Communication

August 2017

Flexible active skin: large reconfigurable arrays of individually addressed shape memory polymer actuators

Nadine Besse, Samuel Rosset, Juan Jose Zarate, and Herbert Shea

N. Besse, Dr. J. J. Zarate, Dr. S. Rosset, Prof. H. Shea

Microsystems for Space Technologies Laboratory (LMTS)

École Polytechnique Fédérale de Lausanne (EPFL)

Rue de la Maladière 71

CH-2000 Neuchâtel,

Switzerland

E-mail: herbert.shea@epfl.ch

Keywords: shape memory polymers; flexible haptic display; soft robotics; flexible actuator

Abstract: A high-resolution flexible active skin with a matrix of $32 \times 24$ individually addressable tactile pixels on a $4 \mathrm{~mm}$ pitch is reported, based on shape memory polymer (SMP) actuators. The intrinsic multistable nature of SMPs, and their more than hundred-fold variation in stiffness over a narrow temperature range, enables dense arrays of actuators exhibiting simultaneously large strokes and high holding forces. The control challenge of addressing a very large number of soft actuators is solved by patterning an array of miniature stretchable heaters on a thin SMP membrane, so that one single pneumatic supply is sufficient to rapidly and selectively reconfigure any or all elements. The device consists of a $40 \mu \mathrm{m}$-thick SMP and sectively rensists of a $40 \mu \mathrm{m}$-thick SMP layer, on which $32 \times 24$ stretchable heaters are integrated, interconnected by a flexible PCB an bonded to a stretchable 3D-printed pneumatic chamber. Each taxel can be individually controlled via row/column addressing, and requires $2.5 \mathrm{~s}$ to latch to a different state. The active skin weighs only $55 \mathrm{~g}$ and is $2 \mathrm{~mm}$ thick. More than $99 \%$ of the 768 taxels are fully functional, with a lifetime in excess of $20 \%$ cycles. This architecture enables applications in haptic displays, active camouflage, biomimetic robots, microfluidics, and new human machine
interfaces.

\section{Main text}

Plants and animals sense their environment to adapt their shape and pose accordingly, often with amazing speed. Anyone who has touched the eyespot at the tip of the tentacles of a snail knows how quickly the gastropod can retract first its tentacles into its body, then most of its body into its shell. Cuttlefish and the Indonesian mimic octopus (Thaumoctopus mimicus) are the best-known examples of animals that change body and skin shape with very high spatial and temporal frequency to replicate a broad range of textures, from smooth, to rippled, or even bumpy. They can thus accurately match the appearance of surrounding animals, plants, rocks or background. ${ }^{[1,2]}$ Much research has been carried out on flexible optical camouflage and purely optical reconfiguration based a wide range of principles, including microfluidics, ${ }^{[3]}$ stretchable light emitting diodes, ${ }^{[4,5]}$ Dielectric Elastomer Actuators (DEAs) ${ }^{[6]}$ and electromechano-chemically responsive elastomers. ${ }^{[7]}$ Yet to date, very little work has been reported on mechanically reconfigurable surfaces with hundreds of independent actuators, for camouflage, communication, haptics displays, or reconfigurable soft robotic systems. The ideal reconfigurable surface would change both appearance and mechanical function.

We present here a novel concept to fully reshape arrays of hundreds of densely packed flexible Shape Memory Polymer (SMP) actuators by synchronizing local Joule heating with a single pressure supply, enabling individual control of all actuators using low voltage signals. We pattern compliant heaters on thin SMP membranes in order to precisely define regions where the stiffness can be locally changed by over two orders of magnitude. By a timely synchronization of the thermal stimuli and the external pressure, each actuator can be independently, reversibly and rapidly latched into several positions (Figure 1). This flexible matrix of latching actuators is highly versatile: in the mechanical domain, it can exert high forces, needed for instance for compliant haptic interfaces, arrays of microfluidic valves, or soft robotic grippers. The deformable surface also controls how electromagnetic waves are reflected, 
leading to applications in camouflage, adaptive optics, and reconfigurable radio-frequency (RF) or millimeter-wave surfaces.

To illustrate the potential of the active skin, we made a high-resolution haptic display because this application is one of the most demanding in terms of force and displacement requirements. Dense arrays of individually addressable actuators for flexible applications are particularly challenging to realize, given the simultaneous requirements on force, displacement, speed, and power consumption for nearly any task where the device must serve a mechanical function. The rapidly growing field of soft robotics generally consists of systems with a very small number of actuators ${ }^{[8]}$ While this is in part because the compliance of soft systems enables shape adaptation with a limited number of transducers, ${ }^{[9]}$ it also reflects the challenge in devising actuation strategies for scalable large matrices of densely packed soft actuators. For instance, most soft manipulators and grippers based on pneumatic actuation, ${ }^{[10,11]}$ granular jamming, ${ }^{[12]}$ DEAs, ${ }^{[13,14]}$ DEA coupled with electro-adhesion, ${ }^{[15]}$ or shape memory alloys ${ }^{[16]}$ have either only one actuator, or several actuators all driven by the same control signal (e.g., a 3-finger gripper driven by a common pressure supply). ${ }^{[17]}$

To allow for a larger number of independent actuators, pneumatic systems can be multiplexed, as shown by Thorsen et al, ${ }^{[18]}$ but this approach has limitations, and scaling to larger number of actuators is unsolved for portable systems. Selective heating of a $65 \times 65$ matrix of stimuli responsive hydrogel has been reported, using an external projector to remotely heat the taxels for actuation. ${ }^{[19]}$ Despite its fast response time, the system exhibited a low force and stroke. Arrays of flexible DEAs were reported by Koo et al as a flexible device that can be wrapped around a finger, but with very short stroke. ${ }^{[20]}$ Addressing large arrays of soft actuators is an unsolved problem.

Phase change materials combine programmable softness and high holding forces. Thermoresponsive SMPs have attracted attention in the last decade for their controllable and reversible stiffness change in response to external thermal stimuli. ${ }^{[21,22]}$ The Young's modulus of SMPs decreases by up to 1000 times over a narrow temperature range when heated above the glass transition temperature $\mathrm{T}_{\mathrm{g}}$, which is generally between $25{ }^{\circ} \mathrm{C}$ and $125{ }^{\circ} \mathrm{C} .{ }^{[23]}$ Recent material from Ren et al exhibits a change from $100 \mathrm{MPa}$ to $0.1 \mathrm{MPa}$ in less than a $10{ }^{\circ} \mathrm{C}$ temperature range. ${ }^{[24]}$ While SMPs have been used for reversible shape change for medical implants ${ }^{[25,26]}$ and for large structures, ${ }^{[27]}$ they have not been implemented for complex shape control as they are generally globally heated, leading to the entire structure changing shape or stiffness.

To avoid the use of external heaters, methods to generate the thermal stimuli directly in the SMP have been studied, notably by rendering the polymer: a) electrically conductive to enable direct Joule heating, ${ }^{[28,29]}$ b) magnetically permeable to allow for contactless induction heating, ${ }^{[30,31]}$ or c) responsive to specific wavelength to enhance radiative heating. ${ }^{[32]}$ Niu et al combined DEAs with SMPs to create an array of Braille-sized bi-stable actuators with up to 240 actuators. ${ }^{[33]}$ Their approach of using an SMP as the elastomer in the DEA elegantly enabled latching in DEAs. ${ }^{[24]}$ For addressable operation, however, a matrix of high-voltage (kV range) switches is required, and independent control of all actuators coupled with selective local heating was not reported.

To demonstrate the ability to locally reshape a flexible surface into any arbitrary topography using only low-voltage signals in a simple row/column architecture, we fabricated the $32 \times 24$ flexible haptic display shown in Figure 1a, with 768 SMP actuators on a $4 \mathrm{~mm}$ pitch. The actuator stroke, pitch, and holding force were chosen to effectively convey dynamic graphical information when explored using the sense of touch. The $32 \times 24$ taxel device consists of: i) a thin SMP membrane with ii) a matrix of carbon-silicone composite stretchable micro-heaters (one heater per actuator), iii) a flexible 4-layer printed circuit board (PCB) for row/column addressing, iv) a 3D-printed stretchable chamber to route the common air pressure to all taxels and, v) two adhesive layers (Figure 1b). The device weighs $55 \mathrm{~g}$ and the active part is $2 \mathrm{~mm}$ 
thick. 757 of the 768 taxels (i.e. $99 \%$ ) are fully functional in the fabricated prototype. The actuator array can be worn as a haptic sleeve with immediate applications in Virtual Reality (VR) and Augmented Reality (AR) scenarios, and for novel Human-Machine Interfaces (HMI). In Figure 1c-f, we display several logos and camouflage patterns on the device. If placed on an autonomous robot, this skin would allow dynamically adapting the robot appearance to the background. Video S1 shows the display refreshing in real time while the device is wrapped around an arm, as well the flexibility of the device.

The central element of our active skin concept is a thin (20 to $100 \mu \mathrm{m}$ thick) SMP membrane, on which a matrix of individually addressed compliant carbon-silicone composite heaters is patterned. This active layer (SMP membrane + heaters) is electrically and mechanically bonded to the flexible PCB to route the Joule heating currents, and then bonded to the stretchable 3Dprinted chamber to distribute a controlled air pressure to the membrane (see Figure 1).

Having one compliant heater per micro-actuator on a thin SMP membrane enables independent, efficient, rapid, and reversible modification of the stiffness of each taxel. By synchronizing the global pneumatic source and local Joule heating, selective, latching, and reversible out-of-plane motion of the active layer is achieved. As illustrated in Figure 2a and shown in Video S1, each taxel starts in the as-fabricated flat state, and can then be deformed into a multitude of temporary inflated or deflated stable shapes, depending on the pressure applied during the actuation cycle. Heating is needed only to switch between stable positions and only the actuators that need to be reconfigured are heated. Heating an actuator without applying any pressure brings it back to its initial ("memory") flat state. When using a pin interface, a negative displacement can be easily transferred into an apparent positive displacement by offsetting the interface (i.e. the maximum negative displacement of the SMP is the "flat" state from the user's perspective, while the flat SMP taxel corresponds to a raised pin). For flexible applications (e.g. camouflage or soft robotics), as we demonstrated with our $32 \times 24$ flexible active skin, one would probably operate without such an interface. For haptic applications, as we demonstrate with our $4 \times 4$ rigid tactile tablet, an interface allows for more effective haptic sensation. For the $3 \mathrm{~mm}$ diameter size we used in this demonstrator, one actuation cycle lasts $5 \mathrm{~s}: 2.5 \mathrm{~s}$ for the moving phase (Joule heating + applied pressure) and $2.5 \mathrm{~s}$ for latching in position at the end of the row-column scan (pressure only + passively cooling). The time scale is given by the heating/cooling time constant of each actuator, and thus depends on taxel size and thickness. The heating and cooling time constants can easily be seen in Video S1 where real-time thermal imaging of the column by column heating is shown alongside visible light imaging showing taxel displacement.

We used a commercially available SMP with a $\mathrm{T}_{\mathrm{g}}$ of $45^{\circ} \mathrm{C}$. We selected this SMP material for its ductility at room temperature (to ease the manufacturing), its high shape recovery/stability (to extend the operating lifetime), and its glass transition temperature of $45^{\circ} \mathrm{C}$ to allow latching in ambient conditions, while requiring only minimal heating to allow for motion ${ }^{[34]}$ The stretchable electrodes are made of carbon black (CB) mixed with a soft polydimethylsiloxane (PDMS). There is a broad range of technologies for stretchable electrodes. ${ }^{[35,36]}$ The carbonsilicone composite we have chosen for the electrodes bonds well to our SMP membrane, can easily be patterned on a large area, ${ }^{[37,38]}$ presents low resistance variation within few tens of percent of strain, ${ }^{[35]}$ and can reliably be used as a heater to over $100^{\circ} \mathrm{C}$. Both the SMP membrane and the $\mathrm{CB} / \mathrm{PDMS}$ electrode are fabricated via blade-casting, with the heater geometry defined via laser ablation, which allows for rapid large-area manufacturing. ${ }^{[36,37]}$ More information can be found in Figure S1.

Given that no information is available on the properties of the SMP membrane when processed as a thin film, and in order to obtain the materials parameters needed to design the active layer (SMP thickness, heater shape and thickness, device dimensions, applied pressure) for different applications, we characterized the mechanical, electrical and thermal properties of the active layer. Specifically, we measured the SMP glass transition temperature, as well as the heat 
capacity, heater resistance, and real and imaginary parts of the Young's modulus as a function of temperature. The SMP membrane used for characterizing these properties was $42.7 \mu \mathrm{m}$ thick and the $\mathrm{CB} / \mathrm{PDMS}$ electrode $23.9 \mu \mathrm{m}$ thick. We have used the material characterization to build a COMSOL Finite Element Analysis (FEA) model of one actuator in order to optimize its geometry. As reported in Figure 3a-b, the FEA simulations match the experiment extremely well in terms of force vs displacement in the cold state $\left(20^{\circ} \mathrm{C}\right)$ and displacement vs applied pressure in the hot state $\left(80^{\circ} \mathrm{C}\right)$ for actuator diameters from $2 \mathrm{~mm}$ to $10 \mathrm{~mm}$. We implemented a non-linear hyperelastic Neo-Hookean model in COMSOL to account for large mechanical deformations. Dynamic mechanical analysis (DMA) was performed on the SMP membrane with and without the CB/PDMS electrode; the temperature dependence of the Young's modulus is reported in Figure 2b and an interpolation of the curve is used as an input parameter for the FEA simulations of Figure 3a-b. In the cold state, we measured a Young's modulus of $1.7 \mathrm{GPa}$ for the bare SMP membrane and 1.1 GPa for the SMP membrane with the CB/PDMS electrode. In the hot state, we obtained $10.7 \mathrm{MPa}$ for the bare SMP membrane and 10.5 MPa for the SMP membrane with CB/PDMS electrode. The datasheet values for the SMP are a Young's modulus of $2.15 \mathrm{GPa}$ for the glassy state and of 1.4 MPa for the rubbery state. The difference between the datasheet and the measured values probably originates from the thickness of the membrane, as the datasheet is for bulk material. The $\mathrm{CB} / \mathrm{PDMS}$ electrode has a resistivity of $0.054 \Omega \mathrm{m}$ and a negative thermal coefficient of resistance (TCR) of $-0.0013{ }^{\circ} \mathrm{C}^{-1}$ (Figure S2c). Differential scanning calorimetry (DSC) was performed to measure the thermal capacity of the materials and to confirm the glass transition temperature obtained with the DMA. The calculated heat capacity curve is reported in Figure S2b. For the bare SMP membrane, the glass transition temperature measured with the DMA and the DSC is $51^{\circ} \mathrm{C}$ and $47^{\circ} \mathrm{C}$, respectively (Figure S2a-b); a value close to the $45^{\circ} \mathrm{C}$ expected from the datasheet. The thermal capacity is $1.2 \mathrm{~J} \mathrm{~g}^{-1}{ }^{\circ} \mathrm{C}^{-1}$ in its cold state and $1.9 \mathrm{~J} \mathrm{~g}^{-1}{ }^{\circ} \mathrm{C}^{-1}$ in its hot state.
For the $32 \times 24$ flexible haptic display, we chose a pitch of $4 \mathrm{~mm}$ (i.e. smaller than a fingertip). The actuator performance is then maximized by designing circular transducers with a $3 \mathrm{~mm}$ diameter; a size and shape which allows for space to electrically and mechanically bond the densely packed array and avoids stress concentration at the clamps. To be easily perceivable by end-users, displacements in the order of $500 \mu \mathrm{m}$ and holding forces over $100 \mathrm{mN}$ are required. ${ }^{[39]}$ Hence, the SMP membrane thickness was set to $40 \mu \mathrm{m}$, a good compromise between force and displacement for a $3 \mathrm{~mm}$ diameter actuator. The FEA simulation predict 400 $\mu \mathrm{m}$ displacement when $300 \mathrm{mbar}$ is applied at $70{ }^{\circ} \mathrm{C}$ and holding forces over $1 \mathrm{~N}$ to move an initially flat membrane down by more than $400 \mu \mathrm{m}$, at room temperature. Determining the optimal membrane thickness is not trivial since the taxel should present both sufficient stroke and force to be perceivable. On the one hand, the thinner the membrane, the larger the displacement, and the lower the holding force. But on the other hand, the thicker the membrane, the smallest the displacement, and the higher the holding force. The thickness of the CB/PDMS compliant heater was chosen to be $25 \mu \mathrm{m}$ in order to have a resistance value between $1.5 \mathrm{k} \Omega$ and $2.5 \mathrm{k} \Omega$. This ensures that less than $30 \mathrm{~V}$ are needed to generate the 200 to $300 \mathrm{~mW}$ required to heat the taxel from $25^{\circ} \mathrm{C}$ to $70^{\circ} \mathrm{C}$ in $1.25 \mathrm{~s}$ (Figure S2d).

We performed aging measurements on one actuator by repeated cycling. As reported in Figure S2e, stable and robust operation was observed when we operated the device continuously for one week (i.e. over $20^{\prime} 000$ cycles). For this test, a cycle lasts $30 \mathrm{~s}$, with $2.5 \mathrm{~s}$ to inflate the taxel, $12.5 \mathrm{~s}$ latched in the "UP" position, $2.5 \mathrm{~s}$ to deflate the taxel and $12.5 \mathrm{~s}$ latched in the "DOWN" position. The timing corresponds to a typical haptic tablet "serious game" scenario where the end-user requires in the order of $10 \mathrm{~s}$ to explore the pattern. The experiment was stopped after 20525 cycles, and the taxel was still operating normally. The small overshoot in displacement corresponds to the thermal expansion/relaxation of the powering phase. The present lifetime 
confirms the robustness of the active layer and could be further improved with active thermal control

Finally, to validate the use of these SMP taxels for haptic applications, perception tests with 15 blindfolded sighted users were conducted to investigate their ability to distinguish graphical information through their sense of touch. We report here the results obtained with the $4 \times 4$ rigid tactile tablet shown in

Figure 4b, which presents similar characteristics as the $32 \times 24$ flexible haptic display; notably the active layer thickness, the actuator pitch and size, and the refresh rate per taxel. The experiment consisted in discriminating among 10 symbols by scanning their finger on the pin interface. Each perception test includes 50 trials, with each symbol displayed randomly. Over $98 \%$ correct pattern recognition was observed in less than $10 \mathrm{~s}$ (Figure 4a). As shown by the $32 \times 24$ flexible haptic display, large-scale and high resolution images can be displayed by selectively moving taxel-by-taxel.

A novel architecture for large array of shape memory polymer micro-actuators has been described and demonstrated. The key innovation consists in patterning compliant heaters on a thin SMP membrane, and synchronizing the local heating of each transducer with a globa pneumatic actuation. This enables to independently and reversibly reshape any array configuration with a simple, highly reproducible, and compact system architecture running at low voltage. As a proof-of-concept of a lightweight and fully reconfigurable soft system with over 750 independently controlled actuators, we reported a $32 \times 24$ flexible haptic display, an important component for emerging human machine interfaces needed for virtual and augmented reality. This actuation concept is extremely versatile and can be applied for instance to soft robotics (giving high holding forces using the programmable stiffness of the SMP), flexible microfluidics valves and micropumps, or reconfigurable RF surfaces for beam-steering or beam-shaping. By replacing the $\mathrm{CB} / \mathrm{PDMS}$ heaters with transparent stretchable electrodes, ${ }^{[40]}$ one could extend our concept to incorporate haptic feedback on flexible screens. 


\section{Experimental section}

Fabrication of the SMP+heater layer: The fabrication process is illustrated in Figure S1. SMP MM4520 pellets (from SMP Technologies Inc., Tokyo, Japan) were dissolved in dimethylformamide (DMF) at a weight ratio $1: 5$, then mixed overnight at $80{ }^{\circ} \mathrm{C}$ with a magnetic stirrer. Next, this SMP+DMF solution was blade-casted on a polyethyleneterephthalate (PET) film with a Teflon AF sacrificial layer using a variable gap applicator (Zehntner ZUA2000) and a film applicator coater (Zehntner ZAA2300). Prior to the casting steps, an oxygen plasma exposure is necessary to activate the surface of the substrate. The SMP membrane was then cured for $2 \mathrm{~h}$ at $80^{\circ} \mathrm{C}$ on a hotplate. The same process was used to blade-cast the CB/PDMS electrode directly on the SMP membrane. The carbon-based composite is a mixture of Ketjenblack EC-600JD (from Akzo Nobel) and Silbione 4305 (Bluestar Silicone) at a weight ratio $1: 10 .^{[37]}$ The $\mathrm{CB} / \mathrm{PDMS}$ electrode is cured for $4 \mathrm{~h}$ at $80^{\circ} \mathrm{C}$. The final layer thickness depends on the applicator gap and the material-to-solvent ratio. We targeted $40 \mu \mathrm{m}$ for the SMP membrane and $25 \mu \mathrm{m}$ for the $\mathrm{CB} / \mathrm{PDMS}$ electrode. The heater and electrical interconnection pattern was defined via laser ablation using a Trotec Speedy $300 .^{[36]}$ Then, the membrane was released and bonded with a silicone adhesive foil (Adhesives Research ARclear 8932EE) to the 4-layer PCB. To ensure good electrical interconnects between the PCB and the heater pads, a silver epoxy (ECCOBOND CE3103WLV) was used. Finally, this active layer and flexible PCB were sealed to the 3D-printed pneumatic chamber using VHB 4905 adhesive from 3M.

Fabrication of the other parts: The $4 \mathrm{x} 4$ pin interface and the rigid pneumatic chamber were 3D-printed in VeroWhite using an Object Connex 500 printer. The stretchable pneumatic chamber was 3D-printed with the same machine, combining TangoBlack for the stretchable parts and VeroWhite for the rigid ones. The PCB was sourced from Beta Layout PCB-Pool with a minimal track width of $150 \mu \mathrm{m}$ and a final thickness of $1.6 \mathrm{~mm}$ for the rigid PCB and of
$0.4 \mathrm{~mm}$ for the flexible PCB. Finally, the aluminum plate was milled using a computer numerical control (CNC) machine.

Acknowledgement

This work was partially supported by the EU within the BlindPAD Project (FP7/2007-2013 Grant Agreement No. 611621). We express our gratitude to O. Gudozhnik, O.A. Araromi, E. Lemaire, S. Schlatter C. A. de Saint-Aubin, A. P. H. Marette and J. Bitterli at the EPFL for helpful discussions. We thank P. Brühlmeier for soldering components on the flexible PCB, and C. Plesse from the University of Cergy-Pontoise for performing the DMA and DSC measurements. We thank our BlindPAD partners at Ateknea Solutions, Geomobile and the Italian Institute of Technology (IIT-RBCS) for their contributions towards the 4x4 rigid tactile display. Finally, we thank all the volunteers who participated in the psychophysical experiment. 
[1] R. Hanlon, Curr. Biol., 2007, 17, 400 .

[2] R. T. Hanlon, L.-A. Conroy and J. W. Forsythe, Biol. J. Linnean Soc. 2008, 93, 23. [3] S. A. Morin, R. F. Shepherd, S. Wai Kwok, A. A. Stokes, A. Nemiroski and G. M. Whitesides, Science, 2012, 337, 828 .

[4] Z. Yu, X. Niu, Z. Liu and Q. Pei, Adv. Mater., 2011, 23, 3989

[5] J. Liang, L. Li, X. Niu, Z. Yu and Q. Pei, Nat. Photon., 2013, 7, 817

[6] J. Rossiter, B. Yap and A. Conn, Bioinspir. Biomim., 2012, 7, 36009

[7] Q. Wang, G. R. Gossweiler, S. L. Craig and X. Zhao, Nat. Commun., 2014, 5, 4899.

[8] S. Bauer, S. Bauer-Gogonea, I. Graz, M. Kaltenbrunner, C. Keplinger and R.

Schwödiauer, Adv. Mater., 2014, 26, 149.

[9] D. Rus and M. T. Tolley, Nature, 2015, 521, 467.

[10] R. F. Shepherd, F. Ilievski, W. Choi, S. A. Morin, A. A. Stokes, A. D. Mazzeo, X. Chen, M. Wang and G. M. Whitesides, PNAS, 2011, 108, 20400.

_[11] M. Wehner, R. L. Truby, D. J. Fitzgerald, B. Mosadegh, G. M. Whitesides, J. A. Lewi and R. J. Wood, Nature, 2016, 536, 451

_[12] E. Brown, N. Rodenberg, J. Amend, A. Mozeika, E. Steltz, M. R. Zakin, H. Lipson and H. M. Jaeger, in Proc. Natl. Acad. Sci., 2010, 107, 18809.

[13] O. A. Araromi, I. Gavrilovich, J. Shintake, S. Rosset, M. Richard, V. Gass and H. R. Shea, IEEE/ASME Trans. Mechatronics, 2015, 20, 438.

[14] G. Kofod, W. Wirges, M. Paajanen and S. Bauer, Appl. Phys. Lett., 2007, 90, 081916. [15] J. Shintake, S. Rosset, B. Schubert, D. Floreano and H. Shea, Adv. Mater., 2016, 28 , 231

_[16] C. Laschi , M. Cianchetti , B. Mazzolai , L. Margheri , M. Follador and P. Dario, $A d v$ Robot., 2012, 26, 709
[17] J. Hughes, U. Culha, F. Giardina, F. Günther and A. Rosendo, Front. Robot. AI, 2016,

3,69

[18] T. Thorsen, S. J. Maerkl and S. R. and Quake, Science, 2002, 298, 580

[19] A. Richter and G. Paschew, Adv. Mater., 2009, 21, 979.

[20] I. M. Koo, K. Jung, J. C. Koo, J.-D. Nam, Y. K. Lee and H. R. Choi, IEEE Trans. Robot., 2008, 24, 549.

[21] M. Behl, A. Lendlein, Mater. Today 2007, 10, 20.

[22] P. T. Mathe, X. Luo, I. A. Rousseau, Annu. Rev. Mater. Res. 2009, 445.

[23] Q. Zhao, H. J Qi, T. Xie, Prog. Polym. Sci. 2015, 49-50, 79.

[24] Z. Ren, W. Hu, C. Liu, S. Li, X. Niu and Q. Pei, Macromolecules, 2016, 49, 134.

[25] J. M. Ortega, W. Small, T. S. Wilson, W. J. Benett, J. M. Loge and D. J. Maitland, IEEE Trans. Biomed. Eng., 2007, 54, 1722

[26] A. A. Sharp, H. V. Panchawagh, A. Ortega, R. ArtaleS. Richardson-Burns S, D. S. Finch, K. Gall, R. L. Mahajan and D. Restrepo, J. Neural Eng., 2006, 3, 23.

[27] Y. Liu, H. Du, L. Liu and J. Leng, Smart Mater. Struct., 2014, 23, 023001.

[28] H. Koerner, G. Price, N. A. Pearce, M. Alexander and R. A. Vaia, Nat. Mater. 2004, 3, 115 .

[29] F. Li, L. Qi, J. Yang, M. Xu, X. Luo and D. Ma, J. Appl. Polym. Sci. 1999, 75, 68.

[30] R. Mohr, K. Kratz, T. Weigel, M. Lucka-Gabor, M. Moneke and A. Lendlein, in Proc. Natl. Acad. Sci. 2006, 103, 3540 .

[31] M. Y. Razzaq, M. Anhalt, L. Frormann and B. Weidenfeller, Mater. Sci. Eng. A 2007, $444,227$.

[32] H. J. Zhang, H. S. Xia, Y. Zhao, J. Mater. Chem. 2012, 22, 845.

[31] X. Niu, X. Yang, P. Brochu, H. Stoyanov, S. Yun, Z. Yu, Z. and Q. Pei, Q. Adv. Mater., 2012, 24,6513 
[32] G. Baer, T. S. Wilson, D. L. Matthews, D. J. Maitland, J. Appl. Polym. Sci. 2007, 103, 3882

[33] S. Rosset, H. R. Shea. Appl. Phys. A 2013, 110, 281.

[34] D. McCoul, W. Hu, M. Gao, V. Mehta, Q. Pei, Adv. Electron. Mater. 2016, 2, 1500407.

[35] O. A. Araromi, S. Rosset, H. R. Shea, ACS Appl. Mater. Interfaces 2015, 7, 18046.

[38] S. Rosset, O. A. Araromi, S. Schlatter, H. R. Shea, J. Vis. Exp. 2016, 108, 53423

[39] F. Vidal-Verdù, M. Hafez, IEEE Trans. Neural Syst. Rehabil. Eng. 2007, 15, 119.

[440] J. Liang, L. Li, D. Chen, T. Hajagos, Z. Ren, S.-Y. Chou, W. Hu, Q. Pei, Nat. Commun. 2015, 6, 7647.

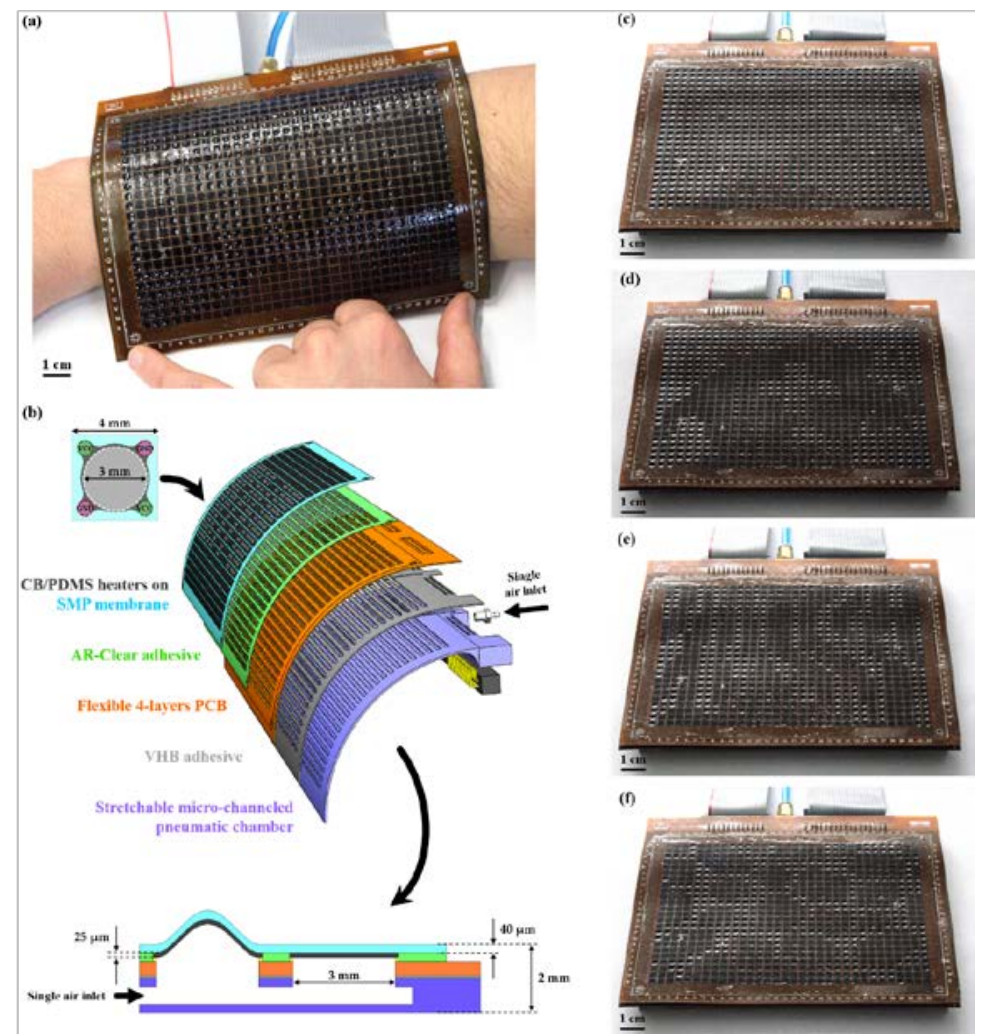

Figure 1. (a) Picture of the flexible $32 \times 24$ taxel active skin in a haptic display sleeve scenario. Each actuator has a diameter of $3 \mathrm{~mm}$, and is placed on a $4 \mathrm{~mm}$ pitch. By patterning compliant heaters on a thin shape memory polymers membrane, we create deformable surfaces where each element is electrically addressed in a row/column architecture, with motion obtained by synchronizing the heating with pressure from one single external pneumatic supply. (b) Schematic exploded view of the $32 \times 24$ flexible haptic display, and cross-section with key dimensions. (c)-(f) Examples of different displayed images for haptics and for camouflage. All taxels latch in both flat and deformed states: zero power is required to hold a pattern. Video S1 shows the patterns being generated. 


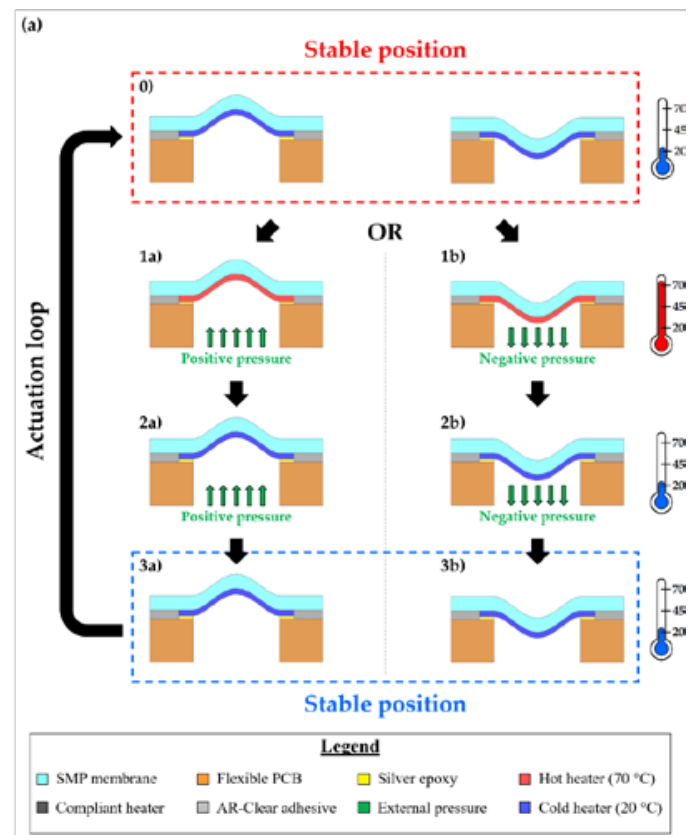

(b)

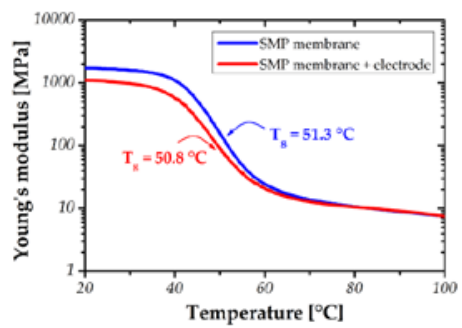

Figure 2. (a) Working principle of the shape memory polymer based taxels. The heater is shown in red when hot $\left(70^{\circ} \mathrm{C}\right.$ ), and in blue when cold $\left(20^{\circ} \mathrm{C}\right.$ ). First, pressure (positive or negative) is applied and the taxels to be actuated are heated using their integrated addressable heaters, reducing their stiffness by more than 100x. Those taxels thus deform. Second, the local heating is turned off and the pressure is kept on to latch the taxel in place as the SMP cools, which takes approximately $1 \mathrm{~s}$. Finally, the pressure is switched off; resulting in a new stable position. The sequence on the left is to raise a taxel, on the right to pull down a taxel. All taxels can be selectively and repeatedly reshaped. Taxels which are not heated remain rigid, and hence do not change position regardless of applied pressure. Video $\mathbf{S} 1$ shows the possible states: flat (no pressure), inflated (positive presure) or deflated (negative pressure). (b) Mesured Young's prosuch , ins electrodes.
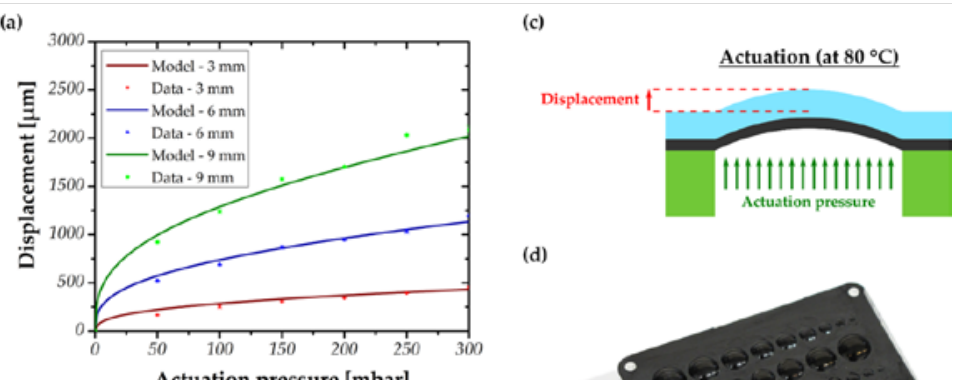

(d)

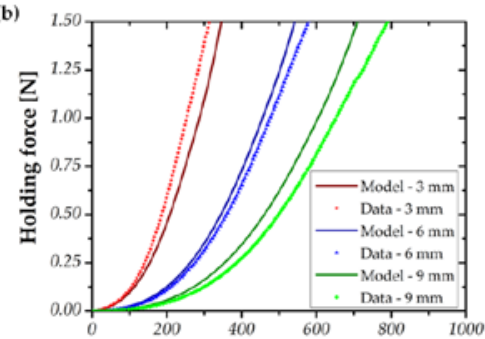

Displacement $[\mu \mathrm{m}]$

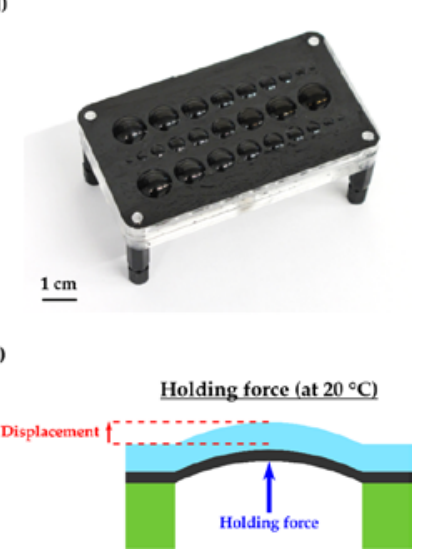

Figure 3. Simulated and measured displacements and holding forces, for SMP actuators with diameters ranging from $3 \mathrm{~mm}$ to $9 \mathrm{~mm}$, showing excellent agreement between model and measured data. (a) Vertical displacement of taxel vs. applied positive pressure at $80^{\circ} \mathrm{C}$. (b) Holding force vs. displacement at $20^{\circ} \mathrm{C}$, starting from a flat state. (c) Schematic illustration of the measured vertical displacement vs. applied positive pressure at $80^{\circ} \mathrm{C}$. (d) Picture of the device used to compare different SMP actuator diameters, with 3 actuators of each size, from 2 $\mathrm{mm}$ to $10 \mathrm{~mm}$ diameter, here all latched in the up state (e) Schematic illustration of the measured holding force vs. displacement at $20^{\circ} \mathrm{C}$, starting from a flat state. 

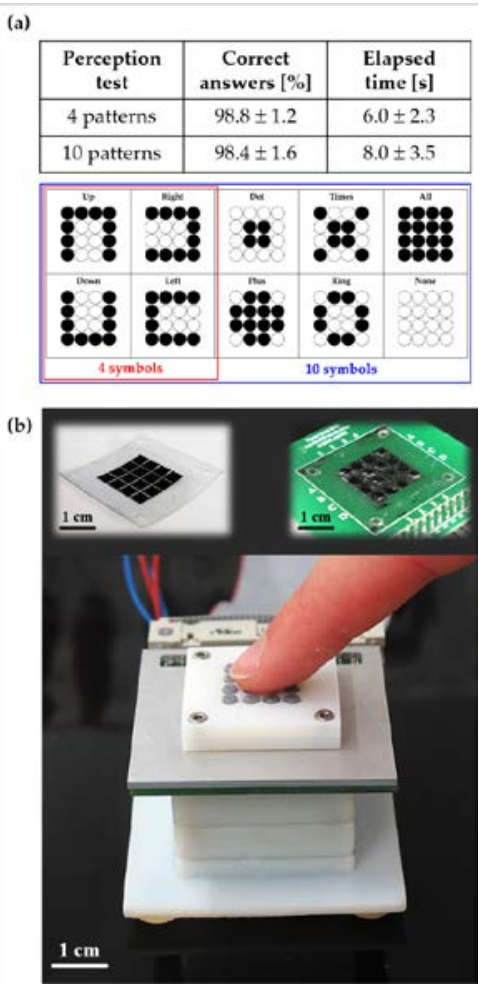

Figure 4. Haptic perception tests with a $4 \times 4$ tactile display where each taxel is driven by one 4 $\mathrm{mm}$ diameter SMP actuator. (a) Table of percentage of symbols correctly identified and of time required for identification for tests on blindfolded volunteers. The symbol database is shown below the table. Nearly $100 \%$ of the symbols are correctly identified even when using the full 10 symbol alphabet. (b) Picture of the $4 \times 4$ tactile display used during the perception full 10 symo alphabet. (b) Picture of the $4 \times 4$ te consisting aluning top a 3 D pred shows the SMP. The to the PCB. The aluminum plate acts as a heat sink and the pin interface prevents direct contact
with the active layer.

\section{TOC summary}

A flexible active skin consisting of a matrix of $32 \times 24$ individually addressable tactile pixels is reported, based on shape memory polymer (SMP) actuators with integrated stretchable heaters. The wearable sleeve weighs only $55 \mathrm{~g}$, is $2 \mathrm{~mm}$ thick and has a $99 \%$ actuator yield. Applications in haptic displays and active camouflage are demonstrated.

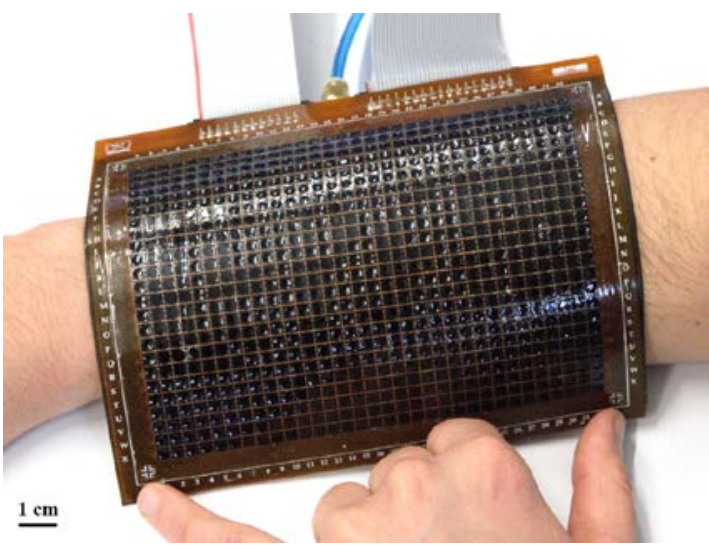


Copyright WILEY-VCH Verlag GmbH \& Co. KGaA, 69469 Weinheim, Germany, 2016

Supporting Information

Video S1. Movie of the flexible $32 \times 24$ latching taxel active skin in a haptic display sleeve scenario. Each actuator has a diameter of $3 \mathrm{~mm}$, and is placed on a $4 \mathrm{~mm}$ pitch. Patterns can be displayed while the device is wrapped around the arm. Real-time visible light and therma images are combined to illustrate the working principle, which consists in synchronizing the local Joule heating of the actuated SMP taxels with a global pneumatic actuation.

Video S1. Movie of the $4 \times 4$ rigid tactile tablet, with the pin interface removed, showing three possible states for the taxels: flat (no pressure), inflated (positive pressure) or deflated (negative pressure). By adding a pin interface on top for the haptic perception tests, displacement over $500 \mu \mathrm{m}$ (i.e. by summing both positive and negative displacement) and holding force over 100 $\mathrm{mN}$ were achieved.

A4-size PET sheet

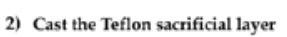

Cast the SMP membrane
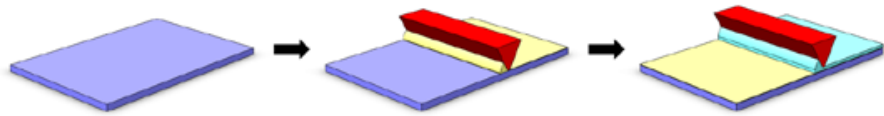

I

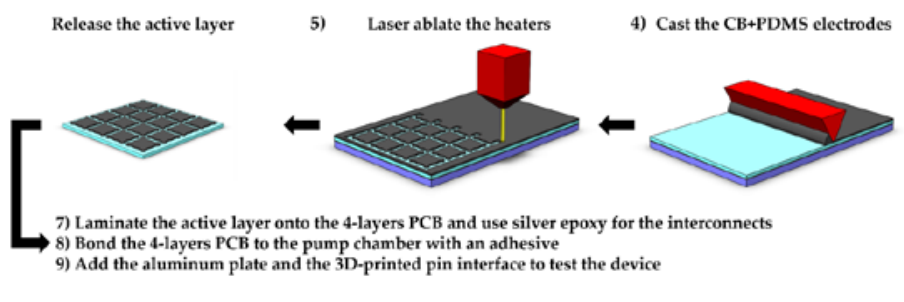

Figure S1. Schematic illustration of the fabrication process. On an A4-size PET sheet, a Teflon AF sacrificial layer, the SMP membrane and the CB/PDMS electrodes are successively bladecasted. Between each step, the solvent is evaporated. The heaters are patterned using laser ablation. Next, the active layer is released form the PET sheet, and is mechanically an electrically interconnected to a 4-layers (rigid or flex) PCB. Finally, the device is assembled and sealed.
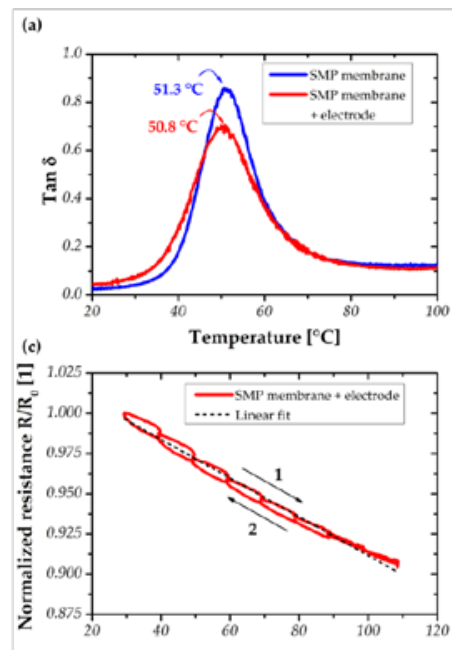

Temperature $I^{\circ} \mathrm{C}$
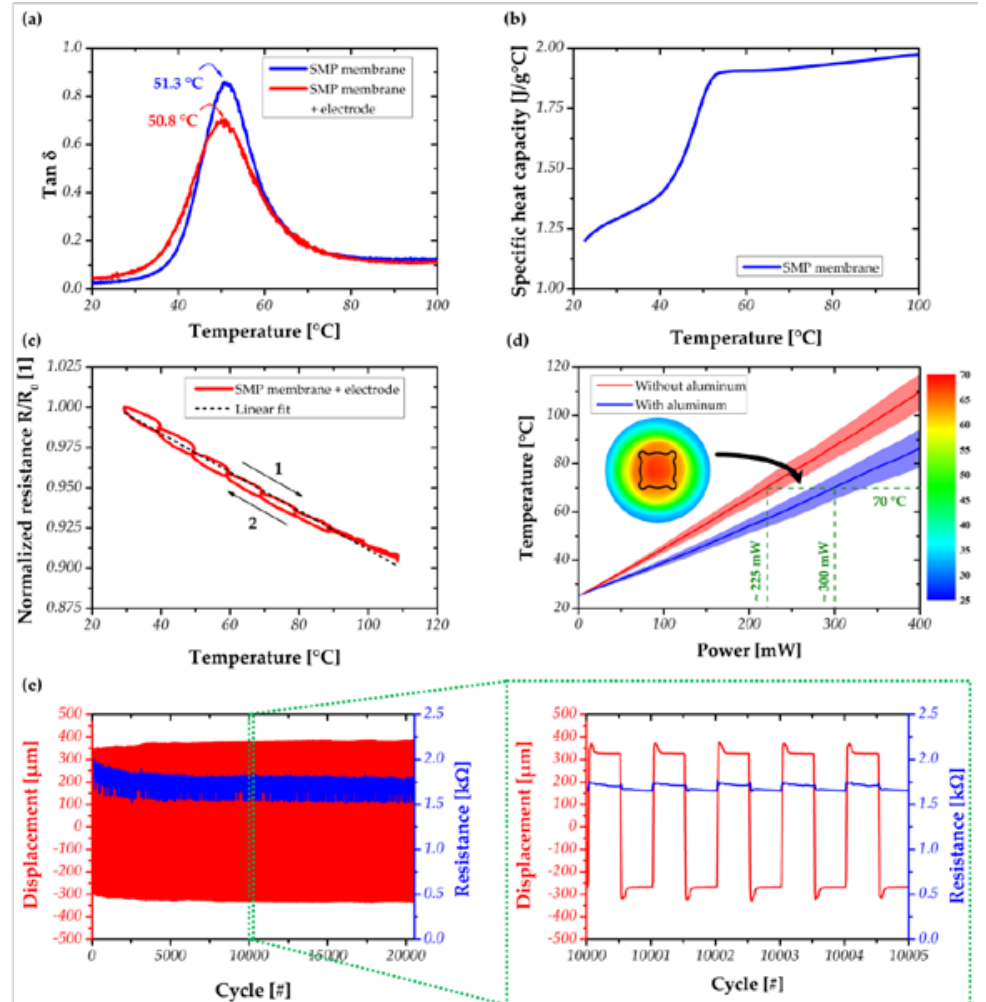

Figure S2. Mechanical, electrical and thermal properties of the SMP membrane and the CB/PDMS heater. (a) Dissipation factor (tangent delta) of the materials showing high losses at the glass transition temperature obtained from a DMA measurement. (b) Heat capacity of the SMP. The glass transition measured with the DSC agrees with the one obtained with the DMA. (c) TCR measurement on a SMP+CB/PDMS strip of $1 \mathrm{~cm} \times 4 \mathrm{~cm}$. The normalized resistance corresponds to the measured resistance value divided by the initial resistance value at $30^{\circ} \mathrm{C}$. (d) Heater tome (d) Her the aluminum plate on tor temp (e) A ing me right. (c) Aging me week (20160 cycles). The displacement and he resistance values are very stable over time. The small initial displacement overshoot at each cycle corresponds to the therma expansion/contraction of the material. 